the concepts. There are also solved problems in the chapters and approximately 120 end-of-chapter exercises. This feature is very helpful for use as a textbook. I am informed by the authors that the Cambridge University Press can provide PowerPoint presentations of the chapters, as well as solved exercises. Consequently, this book should be of great benefit for undergraduate and graduate students in both mechanical engineering and materials engineering who wish to delve into this fascinating subject. Additionally, it is also a valuable reference for researchers in the rapidly expanding fields of the science of biological, bioinspired, and biomaterials. Cambridge University Press is keeping the price at USD\$99 (\$79 for MRS Members), making the book affordable to young scholars.

Reviewer: Robert O. Ritchie is the H.T. \& Jessie Chua Distinguished Professor of Engineering at the University of California-Berkeley, USA.

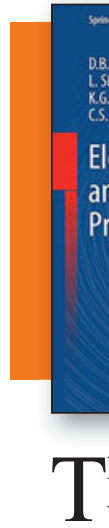

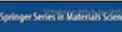

D.8. Srivechmukh

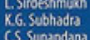

Electrical, Electronic

and Magnetic

Properties of Solids

\section{Properties of Solids}

Electrical, Electronic and Magnetic

D.B. Sirdeshmukh, L. Sirdeshmukh,

K.G. Subhadra, and C.S. Sunandana

Springer, 2014

501 pages, $\$ 179.00$

ISBN 978-3-319-09984-2
Pippard experimental setup are discussed to explain how physical properties are experimentally measured. Basics about semiconductors, including physical properties and their different types, are discussed from a quantum mechanical perspective in the sixth chapter.

Applications of semiconducting devices such as diodes, transistors, and metal oxide semiconductor field-effect transistors, as well as experimental material preparation techniques are very well discussed in the seventh chapter. Different types of magnetism, the associated theory, and the experimental methods to measure magnetic properties are elaborately discussed in the eighth and ninth chapters. An explanation about concepts of domains at the end of the ninth chapter is very interesting, and will give the readers a very clear idea about what happens in magnetic materials in the presence of a magnetic field. In chapter 10, with the help of crystallography and neutron diffraction, different magnetic structures are discussed. Basic components of a neutron diffractometer, an early neutron experimental device (chopper), and a modern-day neutron reactor and their functionalities are pictorially demonstrated. Chapter 11 is dedicated to explaining the fundamentals, theory, and applications of magnetic resonance with the help of experimental tools. Basics and experimental techniques of fast ion conductors with a few example materials are discussed in chapter 12. This chapter will be very interesting for students to learn about the battery and fuel-cell field. The final chapter is dedicated to explain the fundamentals and different types of superconductors along with their applications.

Illustrations in this book that explain the models are very useful to understand concepts. The pictorial representation of random motion of electrons (chapter 2), band filling of electrons at $\mathrm{T}=0 \mathrm{~K}$ (chapter 3), and what happens to the $p-n$ junction when it is biased (chapter 7) are a few examples of clarifying concepts with clear illustrations. Regarding references, the authors might have included more, especially recent ones. In summary, the fundamentals of electrical, electronic, and magnetic materials are well discussed in this book. I strongly recommend this book to all undergraduate and postgraduate students interested in solidstate physics.

Reviewer: K. Kamala Bharathi of the National Institute of Standards and Technology/University of Maryland, USA. such as the cyclotron resonator and the

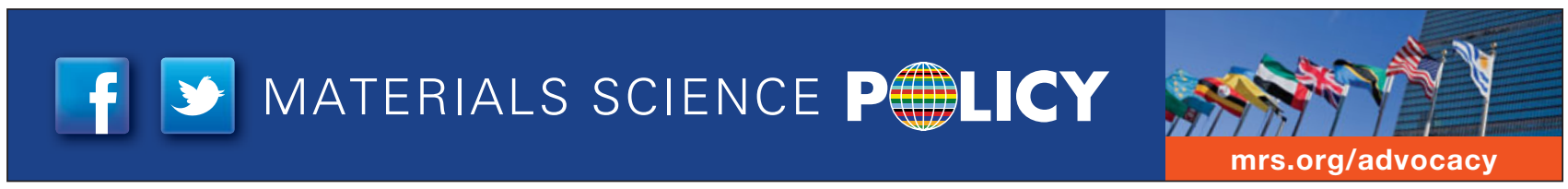

